\title{
Effective temperature scale and bolometric corrections from 2MASS photometry ${ }^{\star}$
}

\author{
E. Masana ${ }^{1,2}$, C. Jordi ${ }^{1,2,3}$, and I. Ribas ${ }^{3,4}$ \\ 1 Departament d'Astronomia i Meteorologia, Universitat de Barcelona, Avda. Diagonal 647, 08028 Barcelona, Spain \\ e-mail: emasana@am.ub.es \\ 2 CER for Astrophysics, Particle Physics and Cosmology, associated with Institut de Ciències de l'Espai-CSIC \\ 3 Institut d'Estudis de Espacials de Catalunya (IEEC). Edif. Nexus, C/Gran Capità 2-4, 08034 Barcelona, Spain \\ ${ }^{4}$ Institut de Ciències de l'Espai, CSIC, Campus UAB, Fac. Ciències, Torre C5 parell, $2^{\mathrm{a}}$ planta, 08193 Bellaterra, Spain
}

Received 9 August 2005 / Accepted 6 January 2006

\section{ABSTRACT}

We present a method to determine effective temperatures, angular semi-diameters and bolometric corrections for population I and II FGK type stars based on $V$ and 2MASS IR photometry. Accurate calibration is accomplished by using a sample of solar analogues, whose average temperature is assumed to be equal to the solar effective temperature of $5777 \mathrm{~K}$. By taking into account all possible sources of error we estimate associated uncertainties to better than $1 \%$ in effective temperature and in the range 1.0-2.5\% in angular semi-diameter for unreddened stars. Comparison of our new temperatures with other determinations extracted from the literature indicates, in general, remarkably good agreement. These results suggest that the effective temperaure scale of FGK stars is currently established with an accuracy better than $0.5 \%-1 \%$. The application of the method to a sample of 10999 dwarfs in the Hipparcos catalogue allows us to define temperature and bolometric correction ( $K$ band) calibrations as a function of $(V-K),[m / H]$ and $\log g$. Bolometric corrections in the $V$ and $K$ bands as a function of $T_{\text {eff }}$, $[m / H]$ and $\log g$ are also given. We provide effective temperatures, angular semi-diameters, radii and bolometric corrections in the $V$ and $K$ bands for the 10999 FGK stars in our sample with the corresponding uncertainties.

Key words. stars: fundamental parameters - stars: late-type - stars: sub-dwarfs - infrared: stars - techniques: photometric - methods: analytical

\section{Introduction}

Effective temperature and luminosity are two fundamental stellar parameters that are crucial for tests of theoretical models of stellar structure and evolution by comparing them with observations. The accuracy in the determination of other stellar properties, such as metallicity, age or radius, hinges on our ability to estimate the effective temperatures and luminosities.

There are several approaches in the literature to compute effective temperature and/or luminosity. Except when applied to the Sun, very few of them are direct methods that permit an empirical measurement of these parameters. Usually, semi-empirical or indirect methods are based on stellar atmosphere models. Among the direct approaches we find the remarkable work by Code et al. (1976), which is based on interferometric measurements of stellar angular semi-diameters $(\theta)$ and total fluxes $\left(F_{\text {bol }}\right)$ at Earth, and the more recent works of Mozurkewich et al. (2003) and Kervella et al. (2004), also based on interferometry. On the other hand, indirect methods

* Full Tables 2 and 3 are only available in electronic form at the CDS via anonymous ftp to cdsarc.u-strasbg.fr (130.79.128.5) or via

http://cdsweb.u-strasbg.fr/cgi-bin/qcat?J/A+A/450/735 are mainly based on the use of photometry, spectroscopy, or a combination of both. In the case of the temperatures, although many of the published calibrations claim to have uncertainties of the order of several tens of degrees, values obtained by different authors can easily have discrepancies of $100 \mathrm{~K}$ or even larger. The reason for such differences must be found somewhere in the methods: atmosphere models, absolute flux calibrations, oscillator strengths, calibration stars, etc.

In this paper we present a semi-empirical method to determine effective temperatures $\left(T_{\text {eff }}\right)$ and bolometric corrections $(B C)$ from $2 \mathrm{MASS}^{1} J H K^{2}$ photometry (Cutri et al. 2003) that is applicable to FGK type stars. As for all others, our method is susceptible to problems derived from the uncertainties in the ingredients mentioned above. However, our approach benefits from two major features: first, it provides a way to evaluate realistic individual uncertainties in $T_{\mathrm{eff}}, \theta$ and luminosity by considering all the involved errors; and second, as it is calibrated to use the 2MASS photometry, it allows the calculation of consistent and homogeneous $T_{\text {eff }}$ and $B C$ for several million stars in the 2MASS catalogue. This paper also provides

\footnotetext{
1 http://www.ipac.caltech.edu/2mass

2 Throughout the paper, $K$ refers to $K_{\mathrm{s}}$ band.
} 
$T_{\text {eff }}$, angular semi-diameters, radii and $B C$ s for $10999 \mathrm{dwarfs}$ and subdwarfs in the Hipparcos catalogue ESA (1997). Such a large sample has allowed us to construct simple parametric calibrations as a function of $(V-K)_{0},[m / H]$ and $\log g$. Note that a preliminary version of the method presented here was successfully applied to the characterization of the properties of planet-hosting stars (Ribas et al. 2003).

The present paper is organized as follows. Section 2 presents the method and explains in detail the procedure to obtain $T_{\text {eff }}$ and angular semi-diameters, including the fitting algorithm, zero point corrections and error estimates. The comparison of our temperatures with several previous works, based on photometric and spectroscopic techniques, is described in Sect. 3. In Sect. 4 we present simple parametric calibrations of $T_{\text {eff }}$ and $B C$ as a function of $(V-K)_{0},[m / H]$ and $\log g$ valid for dwarf and subdwarf stars. The sample of 10999 stars used to build the calibrations is also described in this section together with a detailed explanation of the different contributors to the final uncertainties. Finally, the results are discussed in Sect. 5 and the conclusions in Sect. 6.

\section{The Spectral Energy Distribution Fit (SEDF) method}

The use of infrared (IR) photometry to determine effective temperatures was initially proposed by Blackwell \& Shallis (1977). Their so-called Infrared Flux Method (IRFM) uses the ratio between the bolometric flux of the star and the monochromatic flux at a given infrared wavelength, both measured at Earth, as the observable quantity. This ratio is then compared with a theoretical estimate derived from stellar atmosphere models to carry out the determination of the effective temperature. The IRFM has been widely used by a number of authors, most noteworthy being the work by Alonso et al. (1995, 1996a,b).

The Spectral Energy Distribution Fit (SEDF) method that we propose here follows a somewhat different approach, namely the fit of the stellar spectral energy distribution from the optical $(V)$ to the IR $(J H K)$ using synthetic photometry computed from stellar atmosphere models. Unlike the Alonso et al. (1996a) implementation of the IRFM, which averages temperatures derived individually for each IR band, our method takes into account the four bands simultaneously. In addition, and also unlike the IRFM, the bolometric flux is not required a priori by the SEDF method but results self-consistently with the temperature. The fitting algorithm (see Sect. 2.2) minimizes the difference between observed and synthetic photometry by tuning the values of the effective temperature and the angular semi-diameter. The $B C$ can be obtained from these two parameters, and then, when the distance to the star is known, the luminosity is computed from the $B C$ and the absolute magnitude in a given photometric band. The uncertainties of the derived parameters $\left(T_{\text {eff }}\right.$, angular semi-diameter and $\left.B C\right)$ are estimated from the errors in the observed and synthetic photometry as well as in the assumed $[\mathrm{m} / H], \log g$ and $A_{V}$.

From a theoretical point of view, the SEDF method could be applied to stars of any spectral type and luminosity class. However, the IR flux becomes very sensitive to metallicity and surface gravity for stars hotter than $8000 \mathrm{~K}$ so that small uncertainties in these parameters translate into large uncertainties in the effective temperature. In such situations the SEDF approach becomes inadequate. At the cold end, the accuracy of stellar atmosphere models limits the use of the method to stars hotter than $4000 \mathrm{~K}$ (molecular opacity plays an important role below this temperature). These limitations restrict the applicability of the SEDF to FGK type stars. Fortunately, these stars are very common in the Galaxy and dominate the content of most survey catalogues. They are crucial for several key astrophysical topics, such as the study of the structure and evolution of the Galaxy, both the disk and the halo, and the characterization of planet-hosting stars, among others.

\subsection{Calculation of synthetic photometry}

The calculation of the synthetic photometry requires a wellcharacterized photometric system, an accurate flux calibration and suitable synthetic spectra. The work by Cohen et al. (2003a,b) provides consistent absolute flux calibrations in both the visible $(V)$ (Landolt system) and IR (2MASS JHK) bands. The calibration given by Cohen et al. is computed from a set of calibrated templates, using the synthetic Kurucz spectrum of Vega of Cohen et al. (1992). In the case of the IR photometry, they consider the transmission of the camera and filters, the detector properties and the Earth's atmosphere characteristics. From the comparison between observed and synthetic photometry for a set of 9 A-type stars and 24 cool giants, the authors infer the need to introduce a zero point offset in the synthetic photometry to match the observed 2MASS photometry: $0.001 \pm 0.005 \mathrm{mag}(J) ;-0.019 \pm 0.007 \mathrm{mag}(H) ; 0.017 \pm$ $0.005 \mathrm{mag}(K)$. The calculation of such values is not exempt from difficulty since the dispersions of the differences between both photometries (synthetic and observed) are of the same magnitude as the zero point offsets themselves.

To compute the syntheric magnitudes we made use of the no-overshoot Kurucz atmosphere model grid (Kurucz 1979) taken from http://kurucz . harvard. edu/grids.html:

$m_{\mathrm{syn}}^{i}\left(T_{\mathrm{eff}}, \log g,[m / H]\right)=2.5 \log \left(\frac{F_{\mathrm{cal}}^{i}}{F_{i}\left(T_{\mathrm{eff}}, \log g,[m / H]\right)}\right)$

where $F_{\text {cal }}^{i}$ is the absolute flux calibration given by Cohen et al. (2003b) (for $m_{\text {cal }}^{i}=0$ ) and $F_{i}\left(T_{\text {eff }}, g,[m / H]\right)$ is the flux in the $i$ band computed from the integration of the model atmosphere convolved with the transmission function (filter, detector and Earth's atmosphere) from Cohen et al. (2003b):

$F_{i}\left(T_{\text {eff }}, \log g,[m / H]\right)=\int_{0}^{\infty} \phi\left(T_{\text {eff }}, \log g,[m / H], \lambda\right) \mathcal{T}_{i}(\lambda) \mathrm{d} \lambda(2)$

where $\phi\left(T_{\mathrm{eff}}, \log g,[M / H], \lambda\right)$ is the flux given by the stellar atmosphere model and $\mathcal{T}_{i}(\lambda)$ the effective transmission function in the $i$ band normalized to a peak value of unity.

\subsection{Fitting algorithm}

The fitting algorithm is based on the minimization of the $\chi^{2}$ function defined from the differences between observed 
(corrected for interstellar extinction) and synthetic VJHK magnitudes, weighted by the corresponding error:

$$
\begin{aligned}
\chi^{2}= & \left(\frac{V-A_{V}-V_{\text {syn }}}{\sigma_{V}}\right)^{2}+\left(\frac{J-A_{J}-J_{\text {syn }}}{\sigma_{J}}\right)^{2} \\
& +\left(\frac{H-A_{H}-H_{\text {syn }}}{\sigma_{H}}\right)^{2}+\left(\frac{K-A_{K}-K_{\text {syn }}}{\sigma_{K}}\right)^{2} .
\end{aligned}
$$

This function depends (via the synthetic photometry) on $T_{\text {eff }}$, $\log g,[m / H]$ and a magnitude difference $\mathcal{A}$, which is the ratio between the synthetic (star's surface) and the observed flux (at Earth) $\left(\mathcal{A}=-2.5 \log F_{\text {star }} / F_{\text {Earth }}\right) . \mathcal{A}$ is directly related to the angular semi-diameter by the following expression:

$\theta=10^{-0.2 \mathcal{A}}$.

Although the synthetic photometry depends implicitly on gravity and metallicity, in practice, the spectral energy distribution in the optical/IR for our range of temperatures is only weakly dependent on these quantities. This makes it possible to obtain accurate temperatures even for stars with poor determinations of $\log g$ and $[m / H]$.

As can be seen, the $\chi^{2}$ function depends also on the interstellar absorption $A_{V}$ (the absorption in the other bands can be computed using the extinction law of Schaifers \& Voigt (1982): $A_{J}=0.30 A_{V}, A_{H}=0.24 A_{V}$ and $\left.A_{K}=0.15 A_{V}\right)$. In principle, it is possible to consider $A_{V}$ as a free parameter. However, the strong correlation between $T_{\text {eff }}$ and $A_{V}$, especially for the hotter stars, decreases the precision in the determination of both parameters, with resulting typical uncertainties of $4 \%$ in $T_{\text {eff }}$ and $0.25 \mathrm{mag}$ in $A_{V}$. Thus, for best performance, $A_{V}$ should only be considered as a free parameter when its value is suspected to be large and no other method for its estimation is available. In general, the best approach is to fix the value of $A_{V}$ in Eq. (3) for instance from the estimation of photometric calibration.

Therefore, the only two adjustable parameters by the SEDF method in the present work are $T_{\text {eff }}$ and $\mathcal{A}$, whereas $\log g$, $[\mathrm{m} / H]$ and $A_{V}$ are fixed parameters. To minimize Eq. (3) with respect to these two parameters we use the LevenbergMarquardt algorithm (Press et al. 1992), which is designed to fit a set of data to a non-linear model. In all our tests, convergence towards the minimum value of $\chi^{2}$ was reached rapidly and unequivocally.

\subsection{Calibration of the SEDF method using solar analogues}

The standard procedure for the calibration of an indirect method to determine effective temperatures is based on the comparison of the results with accurate temperatures from $d i$ rect methods for a set of stars. In this way, the list of stars with empirical effective temperatures and angular semi-diameters from Code et al. (1976) has been widely used for calibration purposes. This list has been recently increased with the works of Mozurkewich et al. (2003) and Kervella et al. (2004). Other authors use well-studied stars, such as the Sun, Vega or Arcturus, to calibrate their methods.

Unfortunately, the few stars with empirical values of $T_{\text {eff }}$ are too bright to have accurate 2MASS photometry and they are of no use to calibrate the SEDF method. As an alternative, we have used the list of photometric solar analogues compiled by Cayrel de Strobel (1996). We assume that, as an ensemble, the average of the effective temperatures of these photometric solar analogues should be equal to the effective temperature of the Sun (i.e., $5777 \mathrm{~K}$ ).

After selecting a subsample of 50 unreddened stars with non-saturated 2MASS photometry from Table 1 of Cayrel de Strobel (1996), we computed their temperatures using the SEDF method. We obtained an average temperature of $5832 \pm 14 \mathrm{~K}$, i.e., $55 \mathrm{~K}$ (or $\sim 1 \%$ ) higher than the solar effective temperature. The same value is obtained if we use the subset of solar "effective temperature analogues" from Table 5 of Cayrel de Strobel. Without a deep analysis of all the ingredients involved, from the stellar atmosphere model to the absolute flux calibration, it is very difficult to assess the reasons for such a difference. However, it seems clear that the temperature scale as obtained from the synthetic photometry alone needs a correction to agree with the average of the solar analogues. From a formal point of view, this correction can be computed from the synthetic photometry that results from forcing a value of $T_{\text {eff }}=5777 \mathrm{~K}$ to the entire sample. After doing so, we replaced the zero points given by Cohen et al. (2003b) (see Sect. 2.1) by the average difference (for each band) between the observed and synthetic photometry computed for the solar analogues. Assuming that there is no offset in the $V$ band, the offsets for the other bands are: $0.027 \pm 0.003 \mathrm{mag}(J) ; 0.075 \pm$ $0.005 \mathrm{mag}(H) ; 0.022 \pm 0.005 \mathrm{mag}(K)$. Both in the case of Cohen et al. (2003a) and in our case, the value of the offset in the $H$ band differs significantly from the offsets in $J$ and $K$. The effective temperatures given by Cayrel de Strobel (1996) have not been used here. We have only used the property of the stars in being classified as solar analogues, and, consequently, we assumed their average temperature to be equal to the solar effective temperature.

In our procedure, we are implicitly assuming that the correction in our temperature scale is just a zero point offset and that no dependence on temperature or metallicity is present. These assumptions are justified a posteriori in Sect. 3, where several comparisons of SEDF temperatures with other photometric and spectroscopic determinations are shown.

The angular semi-diameters computed from Eq. (4) were used to check the consistency of the new zero points in our temperature scale. These angular semi-diameters were compared with the direct values compiled in the CHARM2 catalogue (Richichi \& Percheron 2005). We restricted the comparison to stars with accurate VLBI or indirect (spectrophotometry) measurements of the semi-diameter. Only 10 of these stars fulfill the conditions for applicability of the SEDF method. Figure 1 shows the comparison of the semi-diameters for these 10 stars. The agreement is excellent, with an average difference $\left(\theta_{\mathrm{dir}}-\theta_{\mathrm{SEDF}}\right)$, weighted with the inverse of the square of the error, equal to $-0.3 \%$ with a s.d. of $4.6 \%$ (see Table 1 ). All the direct values used in the comparison correspond to a uniform stellar disk. A crude comparison of both uniform disk and limb darkened values for about $1600 \mathrm{~F}, \mathrm{G}$ and $\mathrm{K}$ stars in the CHARM2 catalogue indicates a $\sim 4 \%$ positive correction for limb darkening, of the same order of the dispersion as 


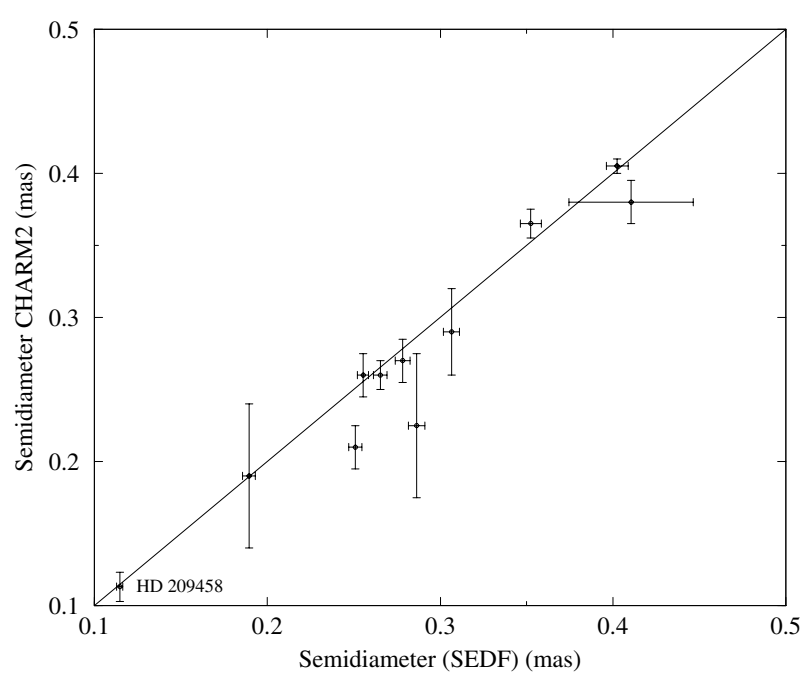

Fig. 1. Comparison of angular semi-diameters computed from the SEDF method (with the new zero point in the temperature scale) and from the CHARM2 catalogue. In the case of HD 209458, the comparison of the semi-diameter is between the SEDF method and an empirical determination from a high-precision transit light curve.

Table 1. Comparison of direct and SEDF angular semi-diameters. $\theta_{\text {dir }}$ are VLBI and spectrophotometric values (Richichi \& Percheron 2005). For HD 209458, $\theta_{\text {dir }}$ is derived from a planetary transit light curve.

\begin{tabular}{lccr}
\hline Star & $\theta_{\text {dir }}(\mathrm{mas})$ & $\theta_{\text {SEDF }}(\mathrm{mas})$ & $\Delta \theta(\%)$ \\
\hline HIP 6702 & $0.190 \pm 0.050$ & $0.189 \pm 0.004$ & 0.5 \\
HIP 8433 & $0.225 \pm 0.050$ & $0.286 \pm 0.005$ & -27.1 \\
HIP 48113 & $0.405 \pm 0.005$ & $0.402 \pm 0.006$ & 0.7 \\
HIP 50786 & $0.260 \pm 0.010$ & $0.265 \pm 0.004$ & -1.9 \\
HIP 51056 & $0.290 \pm 0.030$ & $0.306 \pm 0.005$ & -5.5 \\
HIP 85365 & $0.380 \pm 0.015$ & $0.410 \pm 0.036$ & -7.9 \\
HIP 91237 & $0.210 \pm 0.015$ & $0.251 \pm 0.004$ & -19.5 \\
HIP 96895 & $0.270 \pm 0.015$ & $0.278 \pm 0.004$ & -3.0 \\
HIP 96901 & $0.260 \pm 0.015$ & $0.255 \pm 0.003$ & 1.9 \\
HIP 113357 & $0.365 \pm 0.010$ & $0.352 \pm 0.006$ & 3.6 \\
HD 209458 & $0.113 \pm 0.010$ & $0.115 \pm 0.002$ & -1.8 \\
\hline
\end{tabular}

the relative differences shown in Table 1 . In addition, we compared the radius of HD 209458 obtained with the Hubble Space Telescope from a high precision planetary transit light curve (Brown et al. 2001) with our estimation from SEDF, obtaining very good agreement: $1.146 \pm 0.050 R_{\odot}$ and $1.160 \pm 0.058 R_{\odot}$, respectively.

\subsection{Error estimation}

One of the features of the SEDF method is that it yields individual uncertainties of both $T_{\text {eff }}$ and $\theta$. The total uncertainty can be calculated by combining the contributions from the spectral energy distribution fit and also from the uncertainties in the fixed parameters, i.e., $\log g,[m / H]$, and $A_{V}$. Assuming null correlation between these different (and, in principle, independent) sources of error, the total uncertainty of the $Y_{k}$ parameter $\left(T_{\text {eff }}\right.$ or $\mathcal{A}$ ) is the quadratic sum of the different error contributions:

$$
\begin{aligned}
\left(\Delta Y_{k}\right)^{2}= & \left(\Delta Y_{k} \mathrm{SEDF}\right)^{2}+\left(\frac{\partial Y_{k}}{\partial[m / H]}\right)^{2}\left(\sigma_{[m / H]}\right)^{2} \\
& +\left(\frac{\partial Y_{k}}{\partial \log g}\right)^{2}\left(\sigma_{\log g}\right)^{2}+\left(\frac{\partial Y_{k}}{\partial A_{V}}\right)^{2}\left(\sigma_{A_{V}}\right)^{2}
\end{aligned}
$$

As mentioned above, the minimization of $\chi^{2}$ is carried out with respect to the four magnitudes $V J H K$, with the parameters $A_{V}$, $[m / H]$ and $\log g$ being held fixed. The first term of the equation $\left(\Delta Y_{k}^{\mathrm{SEDF}}\right)$ is the error in the parameter $Y_{k}$ coming from the fit to the spectral energy distribution, which is computed from the covariance matrix $\left(\Delta Y_{k}^{\mathrm{SEDF}} \equiv \sqrt{C_{k k}}\right)$. The derivatives in the other three terms of the equation are determined numerically:

$\frac{\partial Y_{k}}{\partial[m / H]} \approx \frac{Y_{k}(+\Delta[m / H])-Y_{k}(-\Delta[m / H])}{2}$

in the case of the metallicity, and in an analogous way for the surface gravity and the interstellar absorption.

The error in $T_{\text {eff }}$ is obtained directly from Eq. (5), whereas the error in $\theta$ must be calculated from the error in the $\mathcal{A}$ parameter:

$\sigma_{\theta}=0.2 \ln 10 \theta \sigma_{\mathcal{A}}$

The adopted values for the errors in the magnitudes $\left(\sigma_{m_{i}}\right)$, metallicity $\left(\sigma_{[M / H]}\right)$ and surface gravity $\left(\sigma_{\log g}\right)$ are discussed in Sect. 4.1.1.

\section{Comparison with other methods}

Five samples of FGK stars with accurate determinations of effective temperatures (both photometric and spectroscopic) were selected from the literature (Alonso et al. 1996a; Ramírez \& Meléndez 2005; Fuhrmann 1998; Santos et al. 2004; Edvardsson et al. 1993) to carry out a comparison with our results. We paid special attention to correcting for the effects of interstellar reddening, which could lead to systematic differences. For the Alonso et al. and Ramírez \& Meléndez samples (the most reddened), interstellar reddening was corrected using the values of $E(B-V)$ given by the authors so that the two temperature estimations would be directly comparable. For the other three samples, composed of stars at closer distances, we restricted our comparisons to unreddened stars. This meant rejecting very few stars from further analysis. Among several papers in the literature, we chose these five samples because they have a minimum of 25 stars with non-saturated 2MASS photometry and the values of $[\mathrm{m} / H]$ and $\log g$ - needed for a consistent comparison - are provided by the authors.

\subsection{Methods based on IR photometry: Alonso et al. (1996a) and Ramírez \& Meléndez (2005)}

The IRFM is the most popular method to compute effective temperatures from IR photometry. The work by Alonso et al. (1996a) is undoubtedly the widest application of the IRFM to FGK stars. The authors computed effective temperatures for 

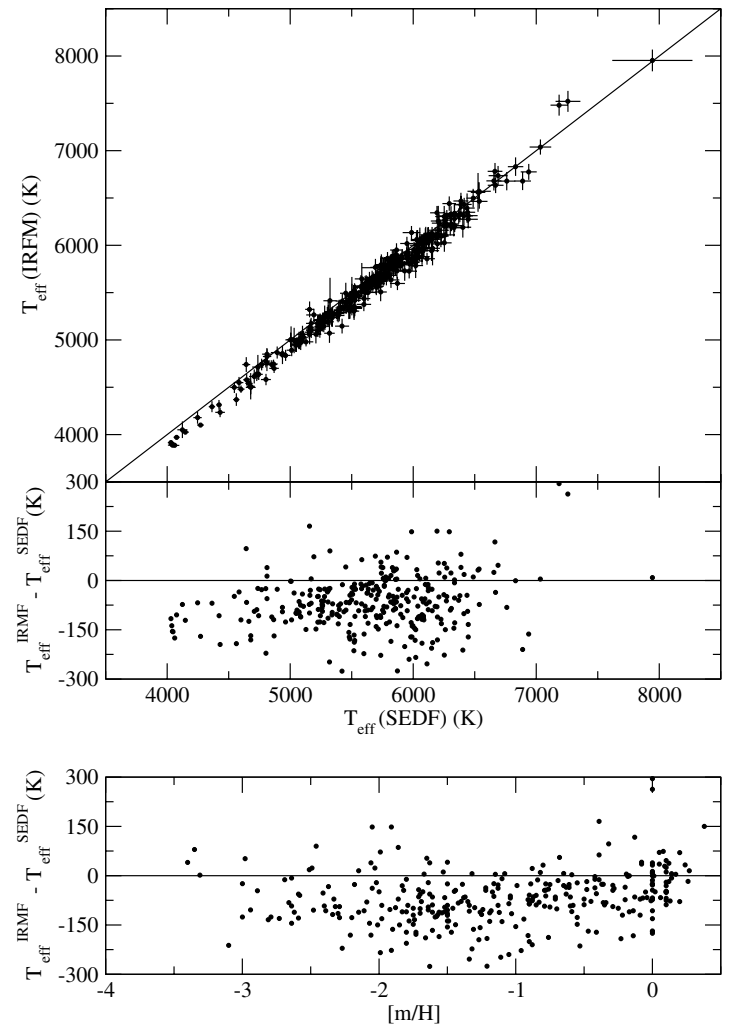

Fig. 2. Comparison of the effective temperatures from the IRFM and the SEDF method for 315 stars in the sample of Alonso et al. (1996a). The bottom panel shows the temperature difference as a function of the metallicity.

462 stars with known interstellar absorption, surface gravity and metallicity. After selecting the stars in the Alonso et al. sample in the range $4000<T_{\text {eff }}<8000 \mathrm{~K}$ and with errors in the 2MASS photometry below $0.05 \mathrm{mag}$, we obtained effective temperatures from the SEDF method for a subset of 315 stars. The comparison between both determinations of $T_{\text {eff }}$ is shown in Fig. 2. The average difference $\Delta T_{\text {eff }}$ (IRFM - SEDF) was found to be $-67 \mathrm{~K}$, with a standard deviation of $81 \mathrm{~K}$. The dependence of this difference on the temperature is not significant: $T_{\mathrm{eff}}^{\mathrm{IRFM}}=1.030 T_{\mathrm{eff}}^{\mathrm{SEDF}}-239 \mathrm{~K}$. As shown in the bottom panel of Fig. 2, there is no dependence of the temperature difference on metallicity.

In a recent work, Ramírez \& Meléndez (2005) recomputed the IRFM temperatures of almost all the stars in Alonso et al. (1996a) using updated input data. According to the authors, the difference between the old and new temperature scales is not significant. They also compare their effective temperatures with some direct determinations. The authors conclude that there is a systematic difference of about $40 \mathrm{~K}$ at solar tem-

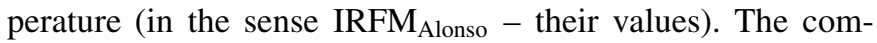
parison between the temperatures of Ramírez \& Meléndez and our determinations is shown in Fig. 3. For 385 stars in common we find $\Delta T_{\text {eff }}$ (IRFM - SEDF) equal to $-58 \mathrm{~K}\left(\sigma_{T_{\text {eff }}}=\right.$ $67 \mathrm{~K})$, and $T_{\text {eff }}^{\mathrm{IRFM}}=1.061 T_{\mathrm{eff}}^{\mathrm{SEDF}}-403 \mathrm{~K}$. Unlike in the case of Alonso et al. (1996a), the dependence of $\Delta T_{\text {eff }}$ on $[m / H]$ is relevant (Fig. 3, bottom panel). For $[m / H]<-2.0$ the temperatures from Ramírez \& Meléndez are clearly hotter than
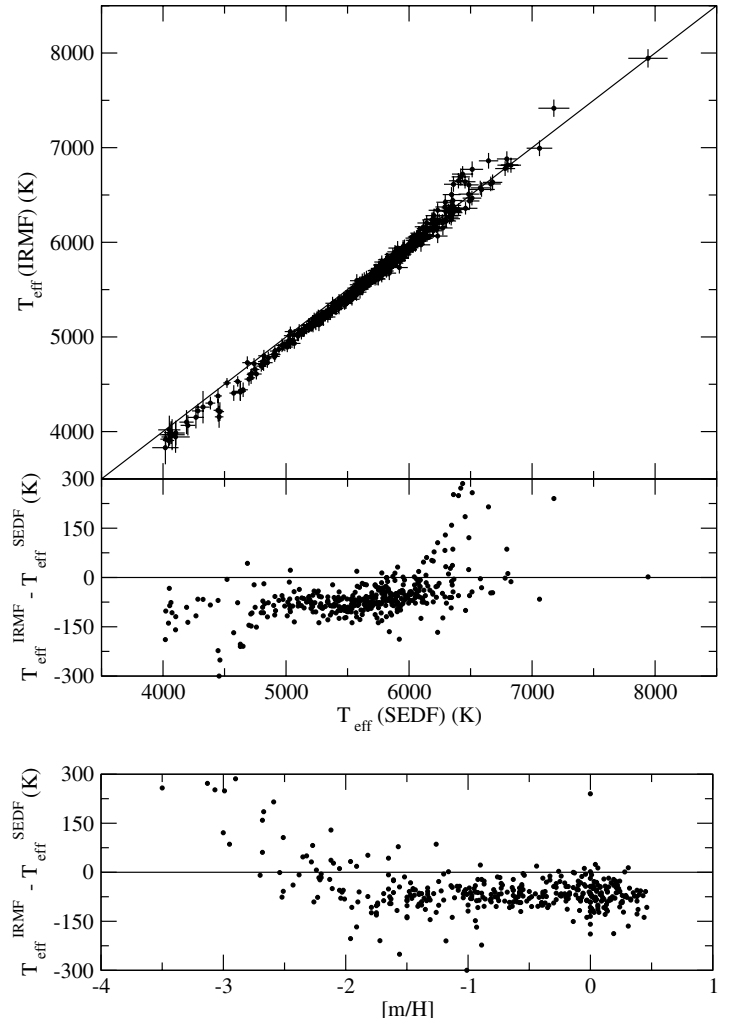

Fig. 3. Comparison of the effective temperatures from the IRFM and the SEDF method for 386 stars in the sample of Ramírez \& Meléndez (2005). The bottom panel shows the temperature difference as a function of the metallicity.

our temperatures. The same trend was found by Charbonnel \& Primas (2005) when comparing their temperatures of 32 halo dwarfs $(-3.5<[\mathrm{Fe} / \mathrm{H}]<-1.0)$ with the values of Ramírez \& Meléndez.

\subsection{Other methods}

In addition to the IRFM, which uses IR photometry, we have also compared the effective temperatures obtained using the SEDF method with other determinations. Two of these (Fuhrmann 1998; and Santos et al. 2004) are spectroscopic works, while in another case (Edvardsson et al. 1993) the temperatures are based on $u v b y-\beta$ photometry. The major problem in the case of spectroscopic determinations is that, in general, they are mostly applied to bright stars, which have poor 2MASS photometry (the 2MASS detectors saturate for stars brighter than $K \approx 4 \mathrm{mag}$ ). This fact reduces the number of stars in the Fuhrmann (1998) and Santos et al. (2004) samples that can be compared with SEDF method.

\subsubsection{Fuhrmann (1998)}

This sample is composed of about 50 nearby $F$ and G stars, both main sequence and subgiants, of the Galactic disk and halo. Effective temperatures were determined from fits to the wings of the Balmer lines. Of those stars, 24 have accurate 2MASS photometry so that reliable SEDF temperatures can 

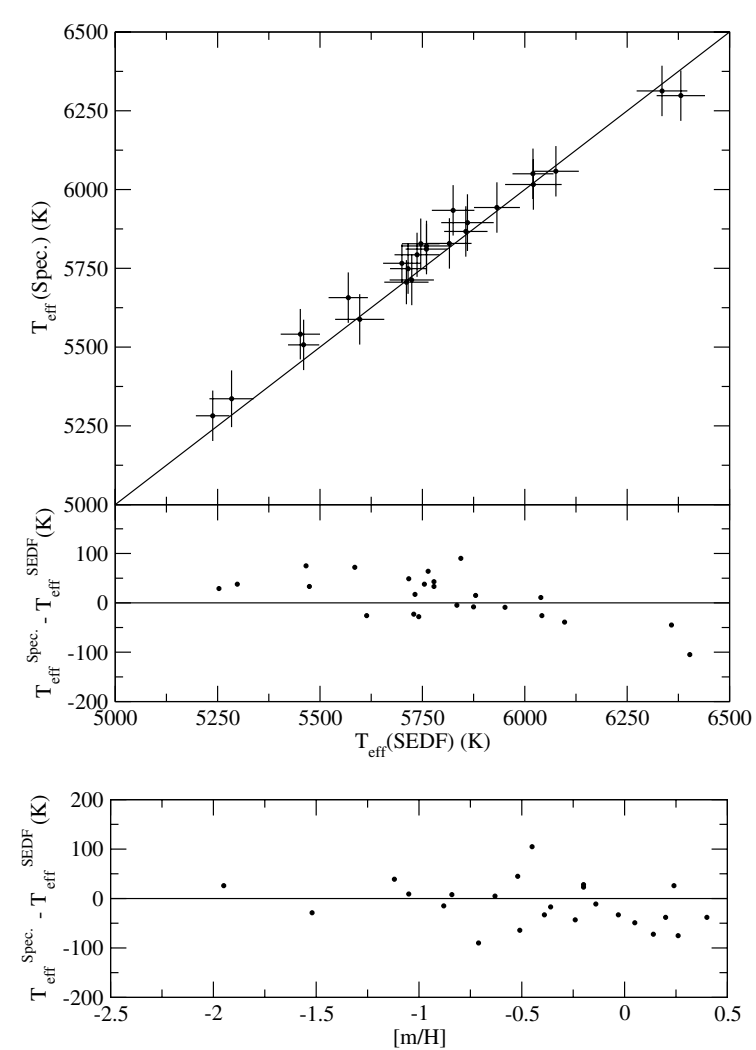

Fig. 4. Comparison of the effective temperatures from fits to Balmer lines and the SEDF method for 24 stars in common with the sample of Fuhrmann (1998). The bottom panel shows the temperature difference as a function of the metallicity.

be derived. The comparison is shown in Fig. 4. The mean average difference $\Delta T_{\text {eff }}$ (Fuhrmann - SEDF) is $12 \mathrm{~K},\left(\sigma_{T_{\text {eff }}}=\right.$ $45 \mathrm{~K})$, with a slight dependence on the temperature: $T_{\mathrm{eff}}^{\text {Fuhrmann }}=$ $0.895 T_{\mathrm{eff}}^{\mathrm{SEDF}}+618 \mathrm{~K}$. No dependence was found between $\Delta T_{\mathrm{eff}}$ and $[m / H]$ (Fig. 4, bottom panel).

\subsubsection{Santos et al. (2004)}

To study the correlation between the metallicity and the probability of a star to host a planet, Santos et al. (2004) obtained spectroscopic temperatures for 139 stars based on the analysis of several iron lines. Effective temperatures for a total of 101 stars in the sample of Santos et al. can be obtained using the SEDF method. In this case, $\Delta T_{\text {eff }}$ (Santos - SEDF) is $28 \mathrm{~K}$, with $\sigma_{T_{\text {eff }}}=68 \mathrm{~K}$, and practically independent of the temperature: $T_{\text {eff }}^{\text {Santos }}=1.053 T_{\text {eff }}^{\mathrm{SEDF}}-270 \mathrm{~K}$ (Fig. 5). There is no dependence of $\Delta T_{\text {eff }}$ with $[\mathrm{m} / H]$ (Fig. 5, bottom panel).

\subsubsection{Edvardsson et al. (1993)}

The sample of Edvardsson et al. is composed of 189 nearby $F$ and $G$ type stars. In contrast with the previous two, the effective temperature is not derived from spectroscopy but from $u v b y-\beta$ photometry. To do so, the authors built a grid of synthetic photometry using the atmosphere models of Gustafsson et al. (1975) and further improved it by adding several new atomic and molecular lines. Effective temperatures
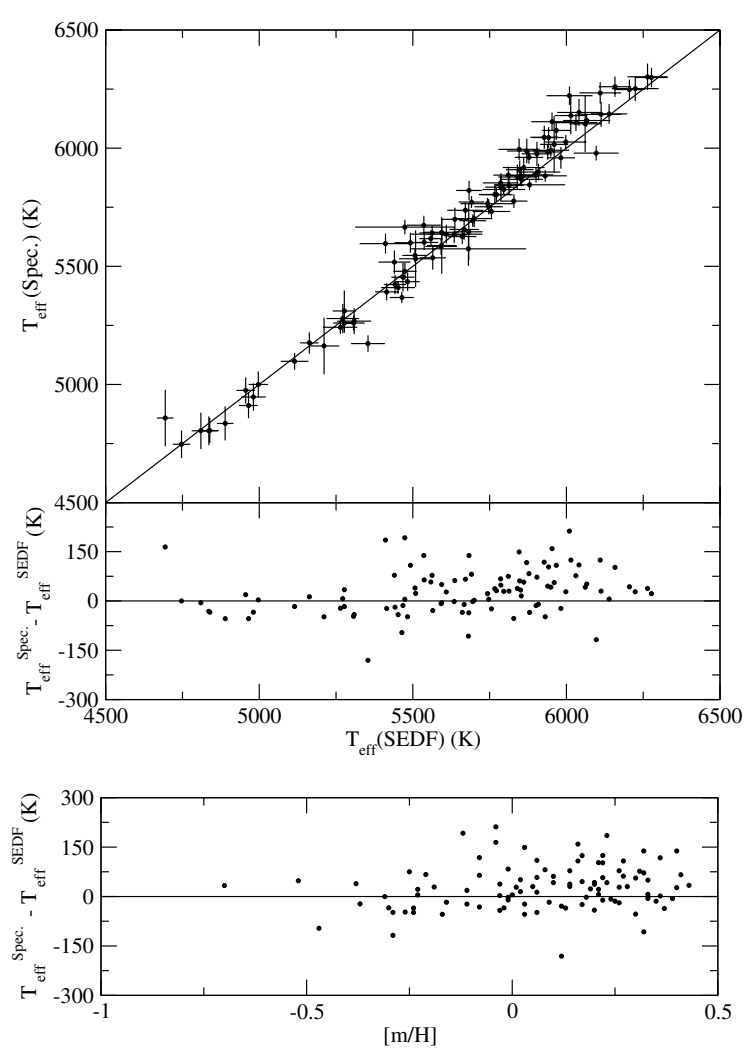

Fig. 5. Comparison of the effective temperatures from iron line fits and the SEDF method for 101 stars in common with the sample of Santos et al. (2004). The bottom panel shows the temperature difference as a function of the metallicity.

for 115 stars in their sample could be derived using the SEDF method. The average difference $\Delta T_{\text {eff }}$ (Edvardsson SEDF) is $10 \mathrm{~K}$, with a dispersion of $70 \mathrm{~K}$ and no dependence on the temperature: $T_{\text {eff }}^{\text {Edvardsson }}=1.006 T_{\mathrm{eff}}^{\mathrm{SEDF}}-27 \mathrm{~K}$ (Fig. 6). As in the two previous cases, the bottom panel of Fig. 6 shows that the temperature difference is not correlated with $[\mathrm{m} / H]$.

\section{Parametric calibrations}

The practical use of the SEDF method as it has been described in Sect. 2 is not straightforward since it requires the calculation of synthetic photometry from stellar atmosphere models and then use of numerical algorithm to minimize the $\chi^{2}$ function. Parametric calibrations (as a function of one or more parameters) may offer a suitable means to estimate reliable effective temperatures in cases where simplicity and speed are to be preferred over the best possible accuracy. In this section we present calibrations for both $T_{\text {eff }}$ and $B C$ as a function of $(V-K)_{0},[m / H]$ and $\log g$. To calculate the calibrations, the SEDF method was applied to a sample of stars in the Hipparcos catalogue, as described below. Note that these calibrations are subject to two limitations with respect to the full SEDF method: first, they are simplifications since not all the available information is used, and second, individual uncertainties cannot be determined. 

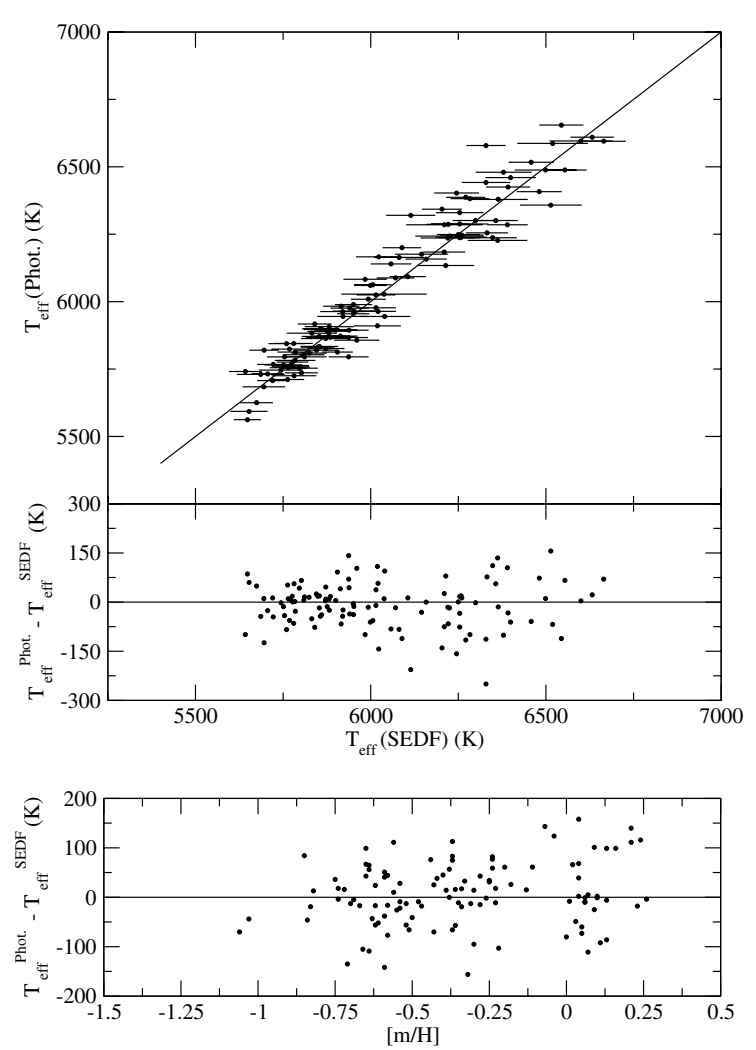

Fig. 6. Comparison of the effective temperatures from photometry and the SEDF method for 115 stars in common with the sample of Edvardsson et al. (1993). The bottom panel shows the temperature difference as a function of the metallicity. The standard deviation for a single star in Edvardsson et al. (1993) is $81 \mathrm{~K}$

\subsection{The stellar sample}

We collected a sample of FGK dwarfs and subdwarfs in the Hipparcos catalogue, and therefore with measured trigonometric parallaxes. Their $V$ magnitudes come mainly from the Hauck \& Mermilliod (1998) catalogue, except for those stars with less than two observations, where we used the Hipparcos catalogue. The entire sample has complete and non-saturated $J H K$ photometry in the 2MASS catalogue. The metallicity was extracted from the compilation of Cayrel de Strobel et al. (2001) or computed from $u v b y-\beta$ photometry - either measured from our own observations or obtained from the Hauck \& Mermilliod (1998) catalogue -, using a slightly revised version of the Schuster \& Nissen (1989) calibration. The range of metallicities covered by the sample is $-3.0 \lesssim[\mathrm{m} / \mathrm{H}] \lesssim$ 0.5 . Values of $\log g$ were computed from $u v b y-\beta$ photometry (Masana 1994; Jordi et al. 1996). Originally, the sample was built to study the structure and kinematics of the disk and halo of the Galaxy (Masana 2004) and a full description including the photometry and a complete set of physical parameters will be provided in a forthcoming paper (Masana et al. 2006).

In spite of the proximity of the stars $(90 \%$ of them are closer than $200 \mathrm{pc}$ ), we computed individual interstellar absorptions from $u v b y-\beta$ photometry and corrected the observed magnitudes. As discussed below, interstellar absorption is one of the most important sources of uncertainty in the $T_{\text {eff }}$ determination.

\subsubsection{Errors}

For our sample, the errors in the magnitudes, metallicity and surface gravity were estimated in the following manner:

- Errors in the VJHK magnitudes: the total error in each magnitude was computed as the quadratic sum of the observational error, the error in the absolute flux calibration and the error in the determination of the interstellar extinction. The first one comes from the photometric catalogues. However, to prevent the underestimation of the error in the $V$ band, usually computed from the average of a few measurements (and with no evaluation of systematics), we have set a minimum error in $V$ equal to $0.015 \mathrm{mag}$. The uncertainties in the absolute flux calibration are given by Cohen et al. (2003a,b) and are in the range 1.5-1.7\% (0.016-0.019 mag), depending on the band. For those stars affected by interstellar reddening, the uncertainty in $A_{V}$ as derived from photometric calibrations based on $u v b y-\beta$ photometry (Jordi et al. 1996) is expected to be of about $0.05 \mathrm{mag}$, or $\sim 1.5 \%$ in $T_{\text {eff }}$.

- Errors in $[\mathrm{m} / \mathrm{H}]$ and $\log g$ : as mentioned above, $[\mathrm{m} / \mathrm{H}]$ was obtained, whenever possible, from spectroscopic measurements, and otherwise we used photometric calibrations, with assigned uncertainties of $0.10 \mathrm{dex}$ and $0.15 \mathrm{dex}$, respectively. We assigned uncertainties of $0.18 \mathrm{dex}$ to $\log g$ values determined from photometric calibrations. The effect on the final effective temperatures due to the uncertainties of both $[m / H]$ and $\log g$ is very small: an error of 0.5 dex in $[m / H]$ has an effect in $T_{\text {eff }}$ of less than $0.5 \%$, whereas the same error in $\log g$ has an effect that ranges between $0 \%$ and $1 \%$, depending on the value of $T_{\text {eff }}$ and $\log g$.

No error was attributed to the flux in the stellar atmosphere models. Comparisons carried out by using other stellar atmosphere models such as those by Castelli et al. (1997) and the NextGen models by Hauschildt et al. (1999) show resulting differences in temperature below $\sim 0.3 \%$ in all cases (Ribas et al. 2003).

An estimation of the final errors in $T_{\text {eff }}$ as function of $T_{\text {eff }}$, $[\mathrm{m} / \mathrm{H}]$ and $\log g$ is shown in Fig. 7. The final error is almost independent of $[\mathrm{m} / \mathrm{H}]$ and $\log g$, but not of $T_{\text {eff. Hotter stars }}$ have greater uncertainties (slightly $>1 \%$ for $T_{\text {eff }}=7500 \mathrm{~K}$ ) than cooler stars $\left(0.6 \%\right.$ for $\left.T_{\text {eff }}=5000 \mathrm{~K}\right)$. In the case of reddened stars, an uncertainty of $0.05 \mathrm{mag}$ in $A_{V}$ can double the error in $T_{\text {eff }}$ compared to the values in Fig. 7. For the angular semi-diameter the behaviour of the errors is very similar to those of the effective temperature, with values for unreddened stars of about 1.0-2.5\%. This means that for Hipparcos stars with good parallaxes, we are able to determine the stellar radii with remarkable uncertainties of about 1.5-5.0\%.

Figure 8 shows the cumulative histograms of the relative errors in effective temperature, angular semi-diameter and radius for the 10999 stars of the sample. As can be seen, about $85 \%$ of the stars have determinations of $T_{\text {eff }}$ better than $1.1 \%$. The relative error in the angular semi-diameter is also better than $1.5 \%$ 

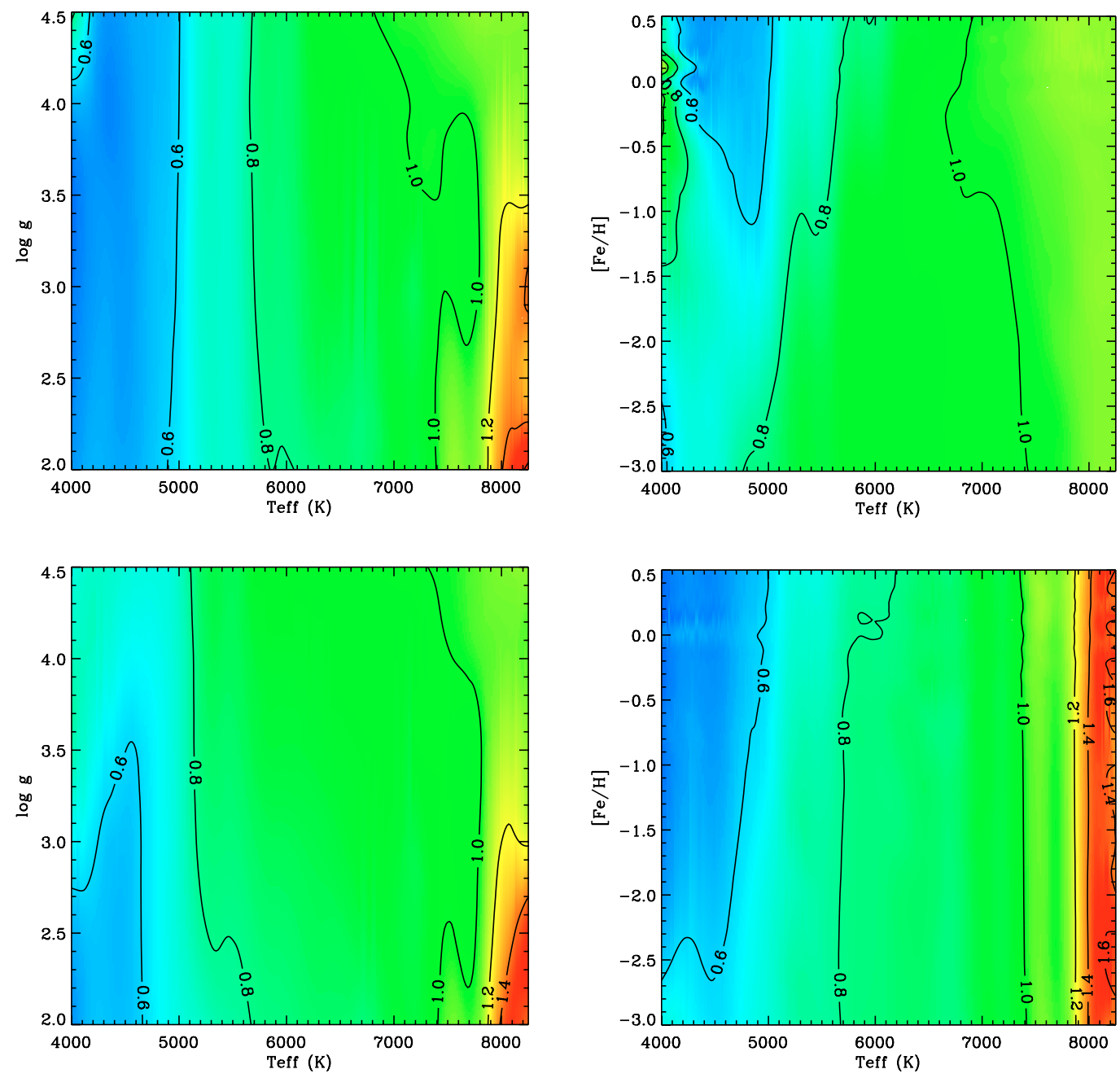

Fig. 7. Relative error (\%) in effective temperature assuming $\sigma_{V J H K}=0.015 \mathrm{mag}, \sigma_{[m / H]}=0.2, \sigma_{\log g}=0.2$ and the error of the absolute flux calibration. Top left: $[\mathrm{m} / \mathrm{H}]=0.0$. Top right: $\log g=4.5$. Bottom left: $[\mathrm{m} / \mathrm{H}]=-2.0$. Bottom right: $\log g=2.0$.

for about $85 \%$ of the stars. In the case of the radii, the main contributor to the error is the uncertainty in the parallax. Even so, $50 \%$ of the stars have radius determinations better than $10 \%$, and $85 \%$ of the stars better than $25 \%$. Most of the stars in our sample are unreddened, thus yielding the best possible accuracy.

Table 2 lists effective temperatures, angular semidiameters, radii and bolometric corrections in the $V$ and $K$ (2MASS) bands with the corresponding uncertainties for the entire sample. Using these values, we calculated simple parametric calibrations of effective temperature and bolometric correction as described below.

\subsection{Effective temperature calibration}

Although the effective temperature for FGK type stars is strongly correlated with the $(V-K)_{0}$ index (see for instance Alonso et al. 1996a), it also depends weakly on the metallicity and surface gravity, as we mentioned in Sect. 2. Therefore, an empirical calibration of $T_{\text {eff }}$ should include terms in all $(V-K)_{0},[m / H]$ and $\log g$. Furthermore, in our case the calibrations were constructed separately in two $(V-K)_{0}$ intervals. Stars departing more than $3 \sigma$ from the fit were rejected. The resulting expressions are:

$$
\begin{aligned}
-\quad 0.35 & <(V-K)_{0}<1.15(4954 \text { stars }): \\
\theta_{\text {eff }}= & 0.5961+0.1567(V-K)_{0}+0.0309(V-K)_{0}^{2} \\
& +0.009[m / H]+0.0022[m / H]^{2} \\
& +0.0021(V-K)_{0}[m / H]-0.0067 \log g \\
\sigma_{\theta_{\text {eff }}} & 0.0028
\end{aligned}
$$

$-1.15 \leq(V-K)_{0}<3.0(5820$ stars $)$ :

$$
\begin{array}{rl}
\theta_{\mathrm{eff}}= & 0.5135+0.2687(V-K)_{0}-0.0174(V-K)_{0}^{2} \\
& +0.0298[\mathrm{~m} / H]-0.0009[\mathrm{~m} / H]^{2} \\
& -0.0184(V-K)_{0}[\mathrm{~m} / H]-0.0028 \log g \\
\sigma_{\theta_{\mathrm{eff}}} & 0.0026
\end{array}
$$

where $\theta_{\text {eff }}=\frac{5040}{T_{\text {eff }}}$. The standard deviation of Eqs. (8) and (9) is about $20 \mathrm{~K}$ and $25 \mathrm{~K}$, respectively. As shown in Fig. 9, there is no residual trend as a function of $(V-K)_{0},[m / H]$ or $\log g$. Equation (8) is aplicable in the range $3.25 \lesssim \log g \lesssim 4.75$ and 

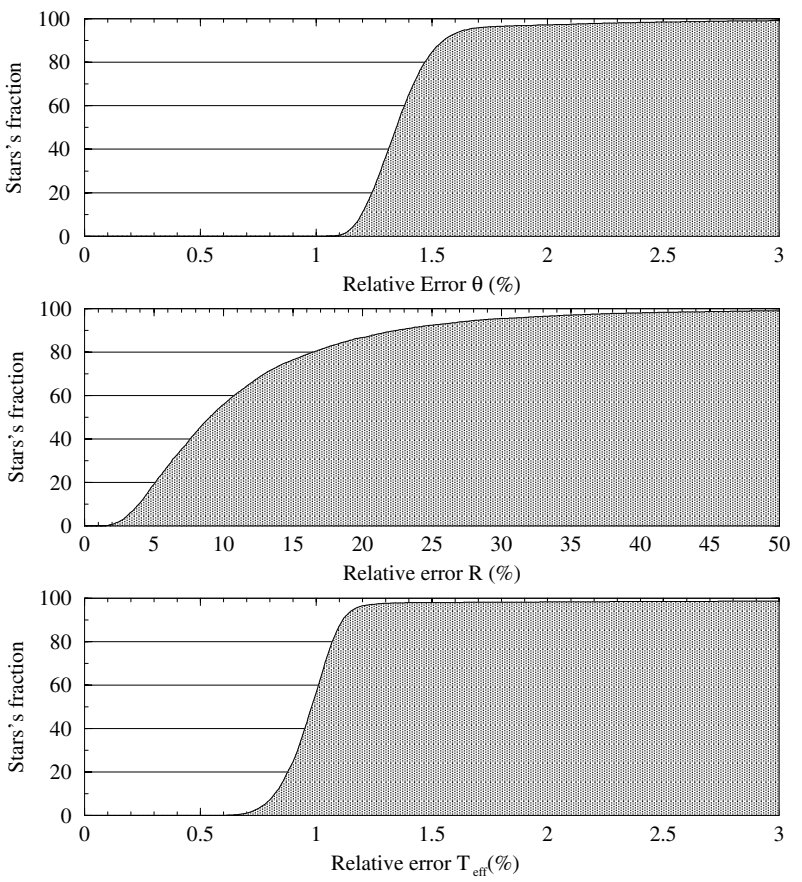

Fig. 8. Cumulative histograms of the relative error in effective temperature, angular semi-diameter and radius for the 10999 stars in the sample.

Eq. (9) in the range $3.75 \lesssim \log g \lesssim 4.75$. Furthermore the calibrations are valid in the ranges of colours and metallicities of the sample:

$$
\begin{aligned}
& -3.0<[m / H]<-1.5 \text { for } 1.0<(V-K)_{0}<2.9 \\
& -1.5 \leq[m / H]<-0.5 \text { for } 0.5<(V-K)_{0}<2.9 \\
& -0.5 \leq[m / H]<0.0 \text { for } 0.4<(V-K)_{0}<3.0 \\
& 0.5 \leq[m / H]<0.5 \text { for } 0.35<(V-K)_{0}<2.8 .
\end{aligned}
$$

While $(\mathrm{V}-\mathrm{K})_{0}$ is an observational quantity and $[\mathrm{m} / \mathrm{H}]$ can be obtained from photometric and/or spectroscopic measurements, a good determination of $\log g$ is usually unavailable for most of the stars. This could severely restrict the applicability of the above calibrations. However, some photometric indexes, such as the Strömgren $\delta c_{1}$ (Crawford 1975; or Olsen 1988), are good surface gravity indicators and, if available, can help to estimate $\log g$. On the other hand, catalogues of spectroscopic metallicities usually provide an estimation of the surface gravity. A crude estimation of $\log g$ can be obtained from MK classification. The error in effective temperature caused by an error in $\log g$ will be:

$\Delta T_{\text {eff }}=\frac{a}{5040} T_{\text {eff }}^{2} \Delta \log g$

where $a$ is the coefficient of the $\log g$ terms in Eqs. (8) and (9). In the worst case, that of the hotter stars, $\Delta T_{\text {eff }}=85 \Delta \log g$. Thus, even if the uncertainty in $\log g$ is as much as $0.5 \mathrm{dex}$, the error induced in $T_{\text {eff }}$ is just $40 \mathrm{~K}$.

The fits for four different metallicities and $\log g=4.5$ together with the stellar sample are shown in Fig. 10. Figure 11
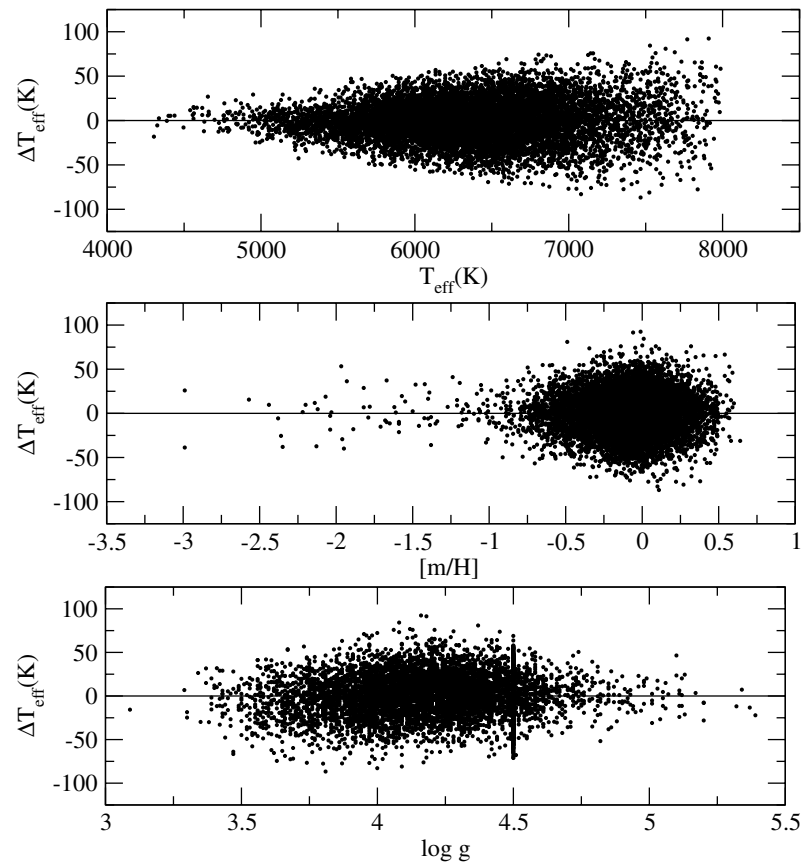

Fig. 9. Residuals of the $T_{\text {eff }}$ fit as a function of effective temperature, metallicity and surface gravity.
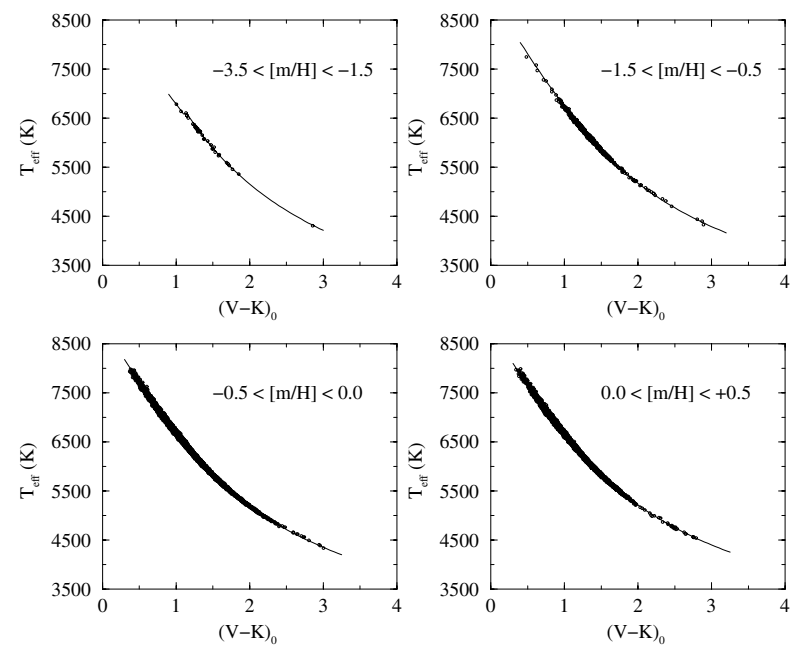

Fig. 10. $T_{\text {eff }}-(V-K)_{0}$ fits for four groups of stars with different metallicities. The empirical relationships correspond to $\log g=4.5$ and $[m / H]=-2.0,-1.0,-0.25$ and +0.25 .

shows the empirical $T_{\text {eff }}-(V-K)_{0}$ relationships as a function of the metallicity.

\subsection{Bolometric correction calibration}

Since the SEDF method provides both effective temperature and angular semi-diameter, it also naturally allows for the determination of the bolometric correction in a specific band. From this, if the distance is known, one can compute the luminosity of the star. The bolometric correction in a given band 


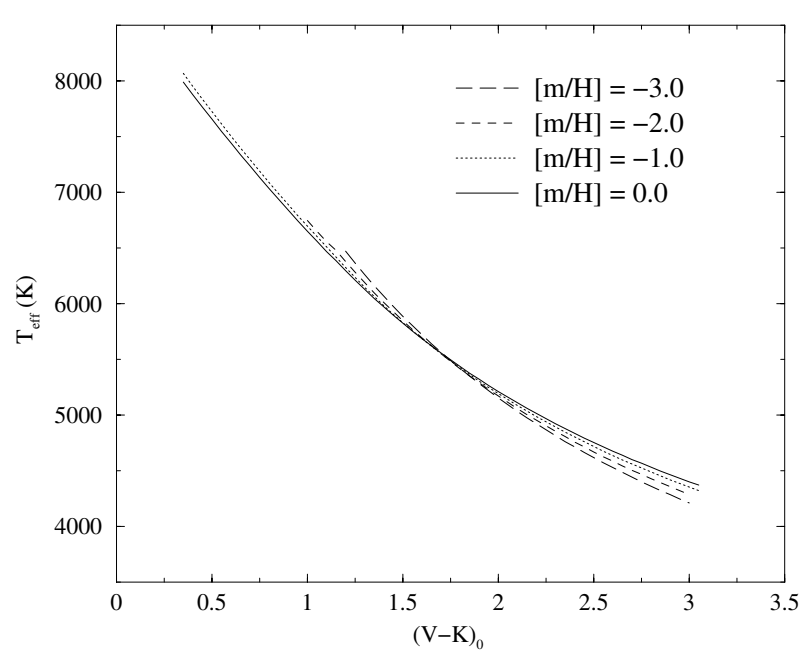

Fig. 11. $T_{\text {eff }}-(V-K)_{0}$ relationships for $\log g=4.5$ and four different metallicities.

is defined as the difference between the bolometric magnitude and the magnitude in that band:

$B C_{i}=M_{\mathrm{bol}}-M_{i}=m_{\mathrm{bol}}-m_{i}$

where $m_{\mathrm{bol}}$ and $m_{i}$ are assumed to be corrected for interstellar reddening. $M_{\text {bol }}$ can be easily expressed as a function of the radius and effective temperature:

$M_{\text {bol }}=-5 \log \frac{R}{R_{\odot}}-10 \log \frac{T_{\text {eff }}}{T_{\text {eff } \odot}}+4.74$

where $R_{\odot}=6.95508 \times 10^{8} \mathrm{~m}$ and $T_{\text {eff } \odot}=5777 \mathrm{~K}$. For the Sun we adopt $V(\odot)=-26.75 \mathrm{mag}$ and $m_{\text {bol }}(\odot)=-26.83 \mathrm{mag}$, and therefore $B C_{V}(\odot)=-0.08(\operatorname{Cox} 2000)$.

Using the definition of the absolute magnitude at a given band $\left(M_{x}=m_{x}+5 \log \pi+5\right)$ and expressing the radius as function of the parallax $(\pi)$ and the angular semi-diameter $(R=\theta / \pi)$, we obtain the following formula for the bolometric correction:

$$
\begin{aligned}
B C_{x} & =M_{\mathrm{bol}}-M_{x} \\
& =-5 \log \left(\mathcal{K} \frac{\theta}{R_{\odot}}\right)-10 \log \frac{T}{T_{\odot}}-0.26-m_{x}
\end{aligned}
$$

where $\mathcal{K}$ is the factor corresponding to the transformation of units. Once the bolometric correction for a band $i$ is known, the bolometric correction for any band $j$ can be determined from:

$B C_{j}=\left(m_{i}-m_{j}\right)+B C_{i}$

The error in the bolometric correction can be expressed as a function of the uncertainties in $T_{\text {eff }}, \theta$ (or $\mathcal{A}$ ) and $m_{i}$, as in Sect. 2.4:

$$
\begin{aligned}
\left(\sigma_{B C_{i}}\right)^{2} & =\left(\frac{5}{\ln 10} \frac{\sigma_{\theta}}{\theta}\right)^{2}+\left(\frac{10}{\ln 10} \frac{\sigma_{T_{\mathrm{eff}}}}{T_{\mathrm{eff}}}\right)^{2}+\left(\sigma_{m_{i}}\right)^{2} \\
& =\left(\sigma_{\mathcal{A}}\right)^{2}+\left(\frac{10}{\ln 10} \frac{\sigma_{T_{\mathrm{eff}}}}{T_{\mathrm{eff}}}\right)^{2}+\left(\sigma_{m_{i}}\right)^{2} .
\end{aligned}
$$
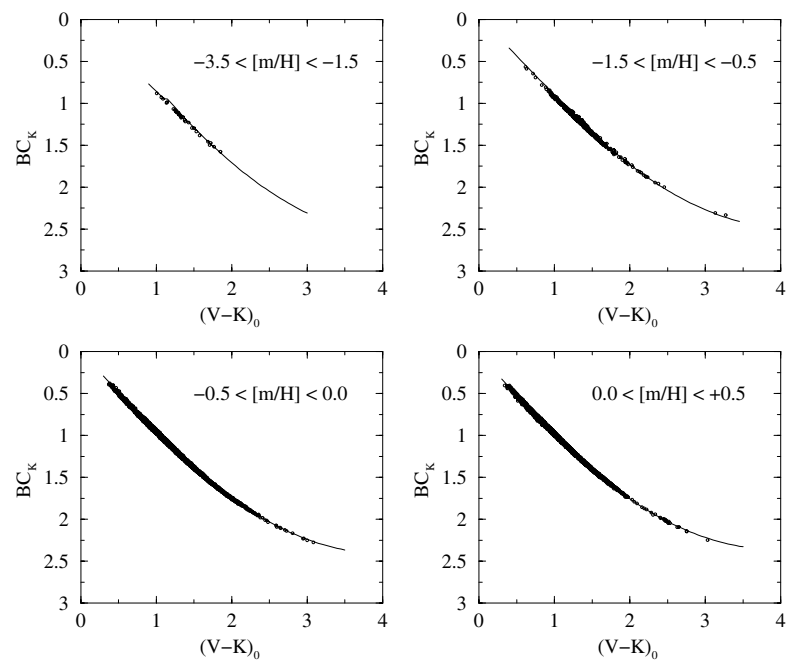

Fig. 12. $B C_{K}-(V-K)_{0}$ fits for four groups of stars with different metallicities. The empirical relationships correspond to $\log g=4.5$ and $[m / H]=-2.0,-1.0,-0.25$ and +0.25 .

The procedure described here was used to compute the bolometric correction in the $K$ (2MASS) band for the stars in our sample. In the same way as for the effective temperature, we calibrated $B C$ as a function of $(V-K)_{0},[m / H]$ and $\log g$ with the following results:

$$
\begin{aligned}
-\quad 0.35 & <(V-K)_{0}<1.15(4906 \text { stars }): \\
B C_{K}= & 0.1275+0.9907(V-K)_{0}-0.0395(V-K)_{0}^{2} \\
& +0.0693[\mathrm{~m} / H]+0.0140[\mathrm{~m} / H]^{2} \\
& +0.0120(V-K)_{0}[\mathrm{~m} / H]-0.0253 \log g \\
\sigma_{B C}= & 0.007 \mathrm{mag}
\end{aligned}
$$$$
\text { - } \quad 1.15 \leq(V-K)_{0}<3.0 \text { (5783 stars): }
$$$$
\begin{aligned}
B C_{K}= & -0.1041+1.2600(V-K)_{0}-0.1570(V-K)_{0}^{2} \\
& +0.1460[m / H]+0.0010[m / H]^{2} \\
& -0.0631(V-K)_{0}[m / H]-0.0079 \log g \\
\sigma_{B C}= & 0.005 \text { mag. }
\end{aligned}
$$

The range of validity of these calibrations is the same as in the case of the effective temperature. The bolometric correction in any band can be obtained from $B C_{K}$ via Eq. (15).

Figure 12 shows the fits for four different metallicities, together with the stars in the sample used to obtain the calibrations. The $B C_{K}-(V-K)_{0}$ relationships as a function of the metallicity are shown in Fig. 13. The calibration is tabulated in Table 3 and compared with the calibrations by Alonso et al. (1995) and Flower (1996) in Fig. 14, showing good agreement.

\section{Discussion}

The procedure described in this paper yields three basic stellar parameters: the best-fitting effective temperature and angular semi-diameter and, from them, the bolometric correction. If the distance is known, $\theta$ can be transformed into the true stellar radius. The accuracies of the parameters for the stars in our 


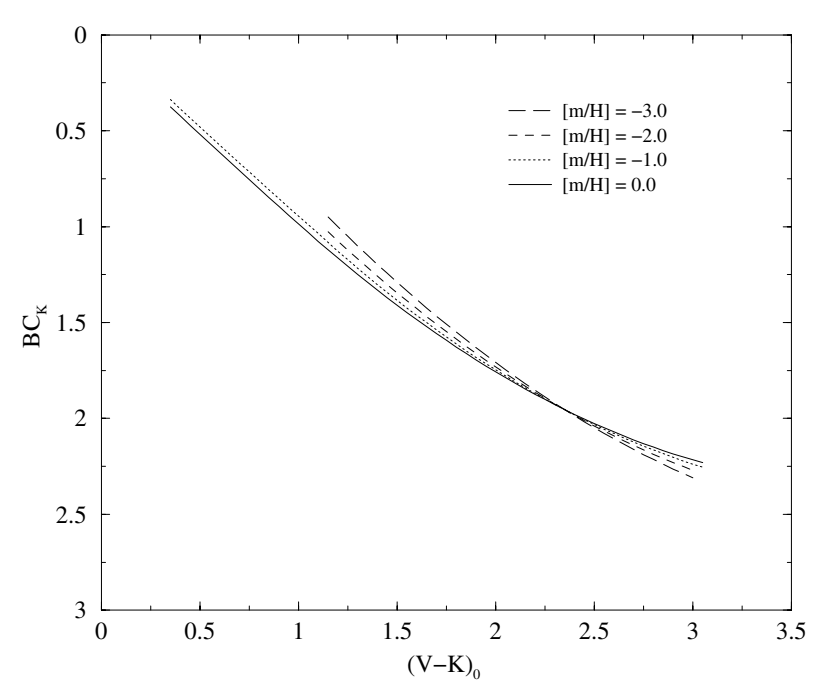

Fig. 13. $B C_{K}-(V-K)_{0}$ relationships for $\log g=4.5$ and four different metallicities.
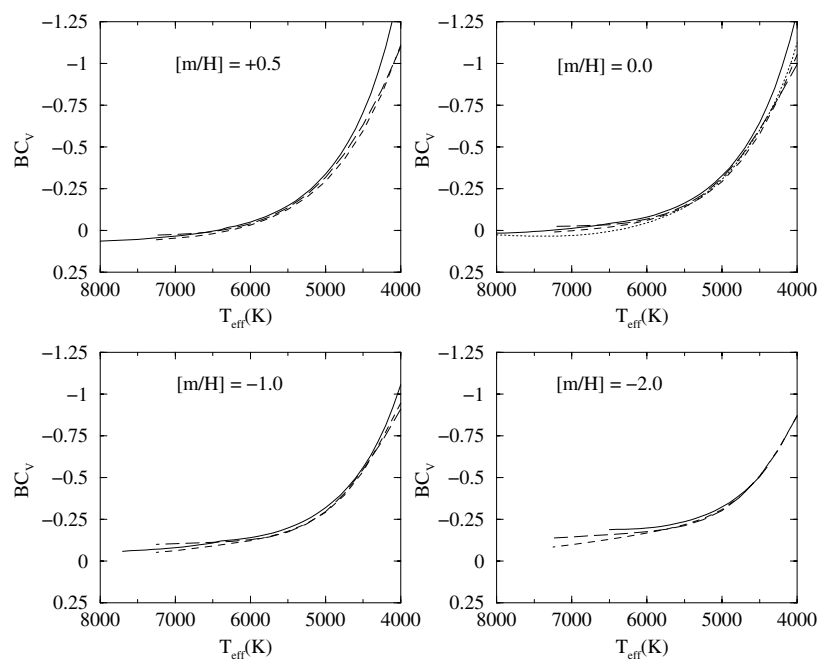

Fig. 14. Comparison between $B C_{V}$ values for $\log g=4.5$ given in Table 3 (solid line) and the values given by Alonso et al. (1995) for $\log g=4$ (dashed line) and $\log g=5$ (long-dashed line). In the panel corresponding to $[m / H]=0.0$, the calibration of Flower (1996) is also shown (dotted line).

sample are $0.5-1.3 \%$ in $T_{\text {eff }}, 1.0-2.5 \%$ in $\theta$ and $0.04-0.08 \mathrm{mag}$ for the $B C$.

Comparisons with other determinations described in Sect. 3 show general good agreement, with differences below $0.5 \sigma$, except for Alonso et al. (1996a) and Ramírez \& Meléndez (2005), where the difference is about $0.8 \sigma$. The use of different atmosphere models and the intrinsic nature of the methods (photometric for Edvardsson et al., Alonso et al., Ramírez \& Meléndez and ours; spectroscopic for Santos et al. and Fuhrmann) can explain in part the small differences. In the case of the IRFM, the main difference between the implementation of both Alonso et al. (1996a) and Ramírez \& Meléndez (2005), and the SEDF method is the absolute flux calibration: Alonso et al. (1994) for the IRFM and Cohen et al. (2003b) for the SEDF. This, together with the use of different versions of the ATLAS9 atmosphere models, is probably the reason for the $\sim 60 \mathrm{~K}$ differential between both implementations of the IRFM and our determination. For Ramírez \& Meléndez (2005) there is a dependence of $\Delta T_{\text {eff }}$ with $[m / H]$ in such a way that the temperature difference (Ramírez \& Meléndez - SEDF) increases abruptly for $[m / H] \lessgtr-2.0$. In all the other cases, the temperature differences are not correlated with $[\mathrm{m} / \mathrm{H}]$.

The most important factor to explain the systematic effects in the effective temperatures computed from different methods is the absolute flux calibration affecting photometric determinations and inaccuracies of model atmospheres (non-LTE effects, 3D effects, treatment on convection, ...) affecting both photometric and spectroscopic determinations. Bohlin \& Gilliland (2004) pointed out a probable $2 \%$ overestimation of the IR flux in the Vega model used by Cohen et al. (2003a). A 2\% shift in absolute flux calibration is equivalent to a difference of about $40 \mathrm{~K}$ in temperature and to a zero point offset in the synthetic photometry of $0.022 \mathrm{mag}$. Such a value would be compatible with our magnitude zero points in Sect. 2.3.

Beyond the internal errors, which in the case of the SEDF take into account the uncertainty in the flux calibration and all other error sources, the comparison with other methods shows that, at present, the systematic errors involved in the determination of effective temperature are of about 20-30 K, equivalent to the $2 \%$ uncertainty in the IR fluxes of Vega claimed by Bohlin \& Gilliland (2004) to be a realistic value.

\section{Conclusions}

We have presented a method (called SEDF) to compute effective temperatures, angular semi-diameters and bolometric corrections from 2MASS photometry. We have adopted an approach based on the fit of the observed VJHK magnitudes using synthetic photometry, and it yields accuracies around $1 \%$ in $T_{\text {eff }}, 2 \%$ in $\theta$, and 0.05 mag in $B C$, in the temperature range $4000-8000 \mathrm{~K}$. A zero point offset was added to the synthetic photometry computed from the Kurucz atmosphere models to link our temperature scale with the Sun's temperature through a sample of solar analogues. From the application to a large sample of FGK Hipparcos dwarfs and subdwarfs, we provide parametric calibrations for both effective temperature and bolometric correction as a function of $(V-K)_{0},[m / H]$ and $\log g$. Note that the method presented here has been selected as one of the main sources of effective temperatures to characterize the primary and secondary targets of the COROT space mission (Baglin et al. 2000). Also, it is being currently implemented as one of the tools offered by the Spanish Virtual Observatory (Solano et al. 2006).

The resulting temperatures have been compared with several photometric and spectroscopic determinations. Although we obtained remarkably good agreement, slight systematic differences with other semi-empirical methods, such as the IRFM, are present. This is probably due to the uncertainties in the absolute flux calibration used by different techniques. It is possible that, in spite of the great effort carried out by Cohen et al. (2003a) and others to construct a consistent absolute flux calibration in both the optical and the IR regions, some problems still remain, which introduce small systematic effects in the temperatures. However, these effects seem to be as small 
as $20-30 \mathrm{~K}$ and could be explained through uncertainties in the IR fluxes of about $2 \%$. Thus, the results presented here strongly suggest that, given the small differences found between methods, the effective temperature scale of FGK stars $(4000-8000 \mathrm{~K})$ is currently established with a net accuracy better than $0.5-1.0 \%$.

Acknowledgements. We are grateful to Dr. Angel Alonso for the suggestion to use solar analogues to calibrate our method. We thank P. Nissen and the anonymous referee for their remarks that helped to improve the paper. We also acknowledge support from the Spanish MCyT through grant AyA2003-07736. I.R. acknowledges support from the Spanish Ministerio de Ciencia y Tecnología through a Ramón y Cajal fellowship. This publication makes use of data products from the Two Micron All Sky Survey, which is a joint project of the University of Massachusetts and the Infrared Processing and Analysis Center/California Institute of Technology, funded by the National Aeronautics and Space Administration and the National Science Foundation.

\section{References}

Alonso, A., Arribas, S., \& Martínez-Roger, C. 1994, A\&AS, 107, 365 Alonso, A., Arribas, S., \& Martínez-Roger, C. 1995, A\&A, 297, 197

Alonso, A., Arribas, S., \& Martínez-Roger, C. 1996a, A\&A, 117, 227 Alonso, A., Arribas, S., \& Martínez-Roger, C. 1996b, A\&A, 313, 873

Baglin, A., Vauclair, G., \& COROT team. 2000, JApA

Blackwell, D. E., \& Shallis, M. J. 1977, MNRAS, 180, 177

Bohlin, R. C., \& Gilliland, R. L. 2004, AJ, 127, 3508

Brown, T. M., Charbonneau, D., Gilliland, R. L., Noyes, R. W., \& Burrows, A. 2001, ApJ, 552, 699

Castelli, F., Gratton, R. G., \& Kurucz, R. L. 1997, A\&A, 318, 841

Cayrel de Strobel, G. 1996, A\&ARv, 7, 243

Cayrel de Strobel, G., Soubiran, C., \& Ralite, N. 2001, A\&A, 373, 159

Charbonnel, C., \& Primas, F. 2005, A\&A, 442, 961

Code, A. D., Davis, J., Bless, R. C., \& Hanbury-Brown, R. 1976, ApJ, 203, 417

Cohen, M., Walker, R. G., Barlow, M. J., \& Deacon, J. R. 1992, AJ, 104,1650
Cohen, M., Megeath, S. T., Hammersley, P. L., Martin-Luis, F., \& Stauffer, J. 2003a, AJ, 125, 2645

Cohen, M., Wheaton, W. A., \& Megeath, S. T. 2003b, AJ, 126, 1090

Cox, A. 2000, Allen's Astrophysical Quantities, Four edition (Springer Verlag N.Y. Inc)

Crawford, D. L. 1975, AJ, 80, 955

Cutri, R. M., Skrutskie, M. F., van Dyk, S., et al. 2003, http://www.ipac.caltech.edu/2mass/releases/allsky/ doc/explsup.html

Edvardsson, B., Andersen, J., Gustafsson, B., et al. 1993, A\&A, 275 , 101

ESA 1997, The Hipparcos and Tycho Catalogues, ESA SP-1200

Flower, P. J. 1996, ApJ, 469, 355

Fuhrmann, K. 1998, A\&A, 338, 161

Gustafsson, B., Bell, R. A., Eriksson, K., \& Nordlund, A. 1975, A\&A, 42,407

Hauck, B., \& Mermilliod, M. 1998, A\&AS, 129, 431

Hauschildt, P. H., Allard, F., \& Baron, E. 1999, ApJ, 512, 377

Jordi, C., Masana, E., Figueras, F., \& Torra, J. 1996, A\&AS, 123, 83

Kervella, P., Thévenin, F., Folco, E. D., \& Ségransan, D. 2004, A\&A, 426, 297

Kurucz, R. L. 1979, AJS, 40, 1

Masana, E. 1994, Master's thesis, Universitat de Barcelona

Masana, E. 2004, Ph.D. Thesis, Universitat de Barcelona

Masana et al. 2006, in preparation

Mozurkewich, D., Armstrong, J. T., Hindsley, R. B., et al. 2003, AJ, 126, 2502

Olsen, E. H. 1988, A\&A, 189, 173

Press, W. H., Teukolsky, S. A., Vetterling, W. T., \& Flannery, B. P. 1992, Numerical Recipes in Fortran, 77 (Cambridge University Press)

Ramírez, I., \& Meléndez, J. 2005, ApJ, 626, 446

Ribas, I., Solano, E., Masana, E., \& Giménez, A. 2003, A\&A, 411, L501

Richichi, A., \& Percheron, I. 2005, A\&A, 434, 1201

Santos, N. C., Israelian, G., \& Mayor, M. 2004, A\&A, 415, 1153

Schaifers, K., \& Voigt, H. H. 1982, Landolt-Börnstein: Numerical data and Functional relationship in Science and Technology, Vol. 2b (Berlin: Springer)

Schuster, W. J., \& Nissen, P. E. 1989, A\&A, 221, 65

Solano et al. 2006, in preparation 